\title{
Fast quantitative optical detection of heat dissipation by surface plasmon polaritons $\uparrow$
}

\author{
Thomas B. Möller, (D) $\ddagger$ Andreas Ganser, $\S \ddagger$ Martina Kratt, Simon Dickreuter, $\uparrow$ \\ Reimar Waitz, || Elke Scheer, (D) * Johannes Boneberg (D) and Paul Leiderer (D)
}

\begin{abstract}
Heat management at the nanoscale is an issue of increasing importance. In optoelectronic devices the transport and decay of plasmons contribute to the dissipation of heat. By comparison of experimental data and simulations we demonstrate that it is possible to gain quantitative information about excitation, propagation and decay of surface plasmon polaritons (SPPs) in a thin gold stripe supported by a silicon membrane. The temperature-dependent optical transmissivity of the membrane is used to determine the temperature distribution around the metal stripe with high spatial and temporal resolution. This method is complementary to techniques where the propagation of SPPs is monitored optically, and provides additional information which is not readily accessible by other means. In particular, we demonstrate that the thermal conductivity of the membrane can also be derived from our analysis. The results presented here show the high potential of this tool for heat management studies in nanoscale devices.
\end{abstract}

\section{Introduction}

Heat dissipation in integrated electronic circuits is an active field of research, because of its importance for the further miniaturisation in nano-electronics. ${ }^{1-3}$ The fundamental aspects of heat flow cover, among others, the recently observed quantum behaviour of thermal conductance, ${ }^{4-6}$ the understanding of the conversion of electrical power via electron-vibron coupling into heat, ${ }^{7,8}$ heating because of scattering at atomic defects in graphene, ${ }^{9}$ local cooling effects, the definition of local temperatures in non-equilibrium systems,${ }^{10,11}$ non-conservative friction forces, ${ }^{12,13}$ as well as bias polarity-dependent and asymmetric heating in molecular junctions. ${ }^{14,15}$ These issues are crucial for the application of nanoscale electronic devices because local heating represents a major source of failure of these devices by electromigration or other thermal destruction. ${ }^{16,17}$ On the other hand, local heating or cooling effects may lend themselves as local energy sources and might give rise to manifold novel transport phenomena. In recent years the conversion of electro-

Department of Physics, University of Konstanz, 78457 Konstanz, Germany. E-mail: elke.scheer@uni-konstanz.de

$\dagger$ Electronic supplementary information (ESI) available. See DOI: 10.1039/ c8nr02489h

$\$$ Both authors contributed equally to the work.

$\S$ Now at: Dep. Mech. Engin., Technical University Munich, 85748 Garching.

ๆNow at: Inst. Appl. Phys., Eberhard Karls Universität Tübingen, 72076 Tübingen.

|| Now at: Rational AG, 86899 Landsberg am Lech. magnetic energy into electrical energy and vice versa in nanooptoelectronic devices has been widely explored and has revealed a palette of hitherto unexpected effects. As an example we mention the observation of photon excitation from nonlinear transport effects in scanning tunnelling microscopy experiments. ${ }^{18-22}$ In many experiments metallic nanoelectrodes act as optical antennae for the local enhancement of the electrical field created by laser irradiation. ${ }^{23-26}$ Localised surface plasmons as well as propagating surface plasmon polaritons (SPPs) ${ }^{27-31}$ represent coupled charge and field effects, and their influence on the optical and charge transport properties has been studied. ${ }^{32-37}$ However, in most cases, the heat transport that goes along with the propagation of SPPs with finite lifetime has been ignored, mainly because of lack of experimental access, although it might have a major impact on the interpretation of nano-optoelectronic experiments. Recently, indirect detection schemes based on conductance changes of atomic contacts and nano-constrictions have been implemented to infer local temperature changes. ${ }^{36,37}$ While these methods have a high spatial resolution, they are time consuming and require a complex calibration for each individual contact under study. To understand the nano- and mesoscale impact of SPPs it is mandatory to study the heat contribution by direct local thermometry, and in equilibrium between the electronic and phononic system, such that a local temperature is well defined. In a previous publication we have shown that it is possible to detect the presence of propagating SPPs by means of their decay into phonons, measuring the resulting temperature increase along the propagation path. ${ }^{38}$ 
In this article we show that the lateral heat flow and the heat dissipation by SPPs in a micron size gold stripe can also be quantitatively determined from an optical inspection of the temperature distribution around the stripe combined with computer simulations. ${ }^{39}$ A thin silicon membrane, used as a substrate for the gold stripe, serves as a medium for obtaining temperature maps with sub- $\mu$ m spatial and sub- $\mu$ s temporal resolution. The comparison between the experiment and simulations yields the efficiency at which incident photons are converted into SPPs and reveals the SPP decay along the propagation path. Moreover, the analysis also provides the thermal conductivity of the thin silicon membrane, which deviates from the value of bulk silicon. These results demonstrate that the technique applied here is a valuable tool for investigating plasmon and heat management in nanostructures.

\section{Results and discussion}

\section{Measurement principle}

We investigate thin Au stripes on a Si membrane supported by a massive Si wafer. ${ }^{40}$ The stripes are patterned with sets of grooves forming line gratings for the excitation of surface plasmons see Fig. 1(a). The gratings are chosen to compensate the momentum mismatch between the SPPs and the exciting photons with wavelength $\lambda=785 \mathrm{~nm} .^{27,37}$ The setup used for our experiments is shown in Fig. 1(b) and consists of an optical microscope to measure the optical transmissivity of the Si membranes with a high spatial resolution and a pulsed light emitting diode for illumination. Since the transmissivity exhibits a pronounced temperature dependence, ${ }^{39}$ the lateral temperature distribution in the membrane can be inferred from the transmitted light, recorded by a digital camera. An example is shown in Fig. 1(c), which represents a temperature map of the central part of a bare membrane when heated with a focused laser beam. The temperature resolution is $0.1 \mathrm{~K}$ and the spatial resolution is limited by the resolution of the microscope to $\sim 400 \mathrm{~nm}$. Time-resolved measurements which show the spreading of heat after pulsed laser excitation can be realised using a pump-probe technique, where the sample is illuminated by the probe light with some delay after the pump laser pulse. With the present setup the time resolution in the temperature measurement is about $100 \mathrm{~ns}$, given by the rise time of the pump heating laser diode and the LED probe light. The details of the measurement procedure have been published in ref. 38 and are given in the Experimental section and the ESI. $\dagger$

\section{Steady-state measurements}

We first describe the observations of the temperature distribution in the Si membrane when the grating in the gold stripe is illuminated continuously on a time scale of seconds or longer. Since the characteristic thermal relaxation time of the sample is in the range of a few microseconds, this setting corresponds to a steady-state situation of the system. Fig. 2 shows the temperature increase around the grating for two orientations of the light polarisation (in Fig. 2(a) parallel to the grooves of the grating; in Fig. 2(b) perpendicular to the grooves of the grating). We label the polarisation perpendicular to the grooves (i.e. parallel to the propagation direction of the SPPs along the stripe) as p-polarisation, and the one per- (a)

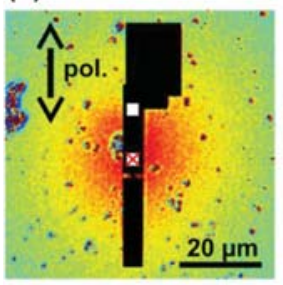

(b)

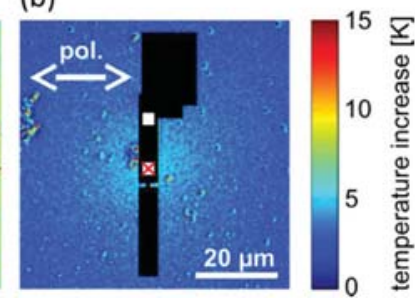

Fig. 2 Temperature maps for $\mathrm{p}$ - and $\mathrm{s}$-polarisation of the incident laser light with the same intensity. In (a), the polarisation is perpendicular, and in (b), the polarisation is parallel to the grating grooves. The area of the gold film used for this sample appears here in black; the position of the grating near the centre of the image is marked by a cross. The irregular dots in the images are artefacts, caused by slight contaminations of the Si membrane.
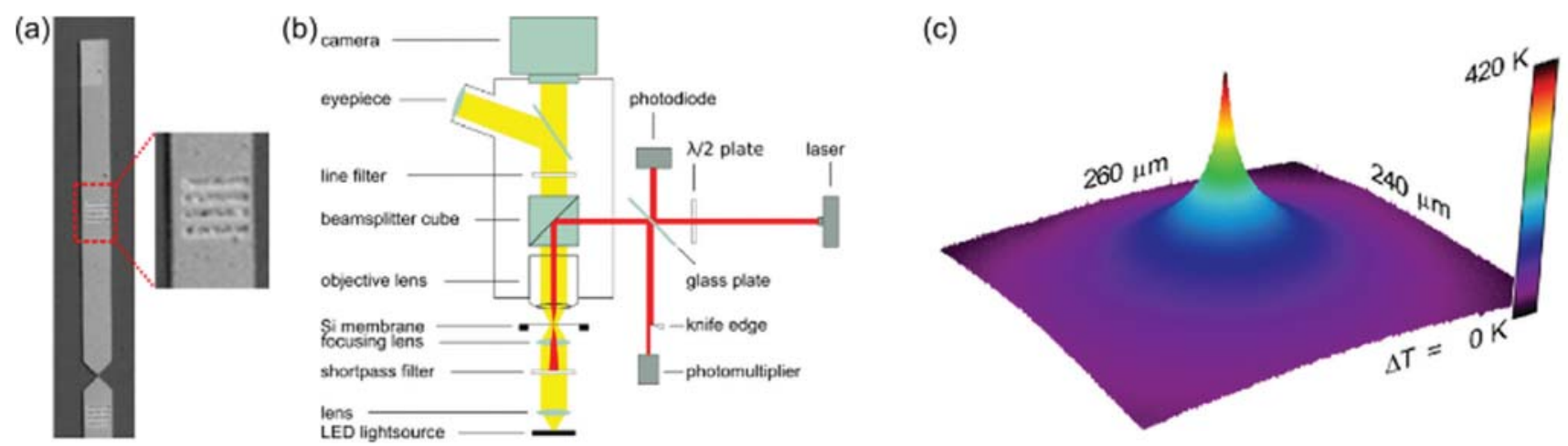

Fig. 1 (a) Typical sample design: gold stripe on a silicon membrane with a grating produced by focused ion beam (FIB) patterning. (b) Schematic optical setup, details can be found in the ESI. $\dagger$ (c) Map of the temperature increase $\Delta T$ in a bare silicon membrane heated in the centre with a focused laser beam. 
pendicular to the stripe as s-polarisation. It is obvious that the temperature increase is distinctly higher for p-polarisation; hence the absorption of the incident laser light by the grating must be much more efficient in Fig. 2(a) than in Fig. 2(b). The difference of nearly a factor of 4 of the temperature increase can be ascribed to resonant plasmonic excitations, which couple more efficiently to the p-polarised light.

We would like to mention that in spite of the clear effect of the polarisation in Fig. 2, this observation is not a proof that propagating SPPs are indeed generated in this case; it only means that p-polarised light is more strongly absorbed by the grating. The time-resolved measurements presented in the next section, however, will provide clear evidence for the propagation of SPPs in the gold stripe.

The temperature maps in Fig. 2 do not only illustrate the big difference in the absorptivities for $\mathrm{p}$ - and s-polarised light, but they also allow one to determine the total power dissipated when the grating is illuminated. This can be done, e.g., by a comparison with simulations like the one shown in Fig. 6. However, we start here from a different, analytical approach, using the fact that the temperature in a locally heated membrane - as shown in Fig. 1(c) - varies as

$$
T(r)=-(P / 2 \pi \kappa d) \ln \left(r / r_{0}\right)+T_{0},
$$

where $P$ is the absorbed input power, $r$ the radial coordinate, $d$ the thickness of the membrane, $\kappa$ the thermal conductivity, and $r_{0}$ and $T_{0}$ are the starting radius and temperature, respectively. ${ }^{39}$ Eqn (1) is valid for distances larger than the size of the heat source and for an isotropic sample. ${ }^{41}$ While in our samples the Si membrane itself is isotropic, some anisotropy in the temperature distribution arises due to the presence of the gold stripe. This is only a small effect, however, as was shown for unpatterned films in ref. 38, and is confirmed in the ESI in Fig. S4. $\dagger$ From the observed temperature gradients in steady state one obtains, using eqn (1), the heat that is flowing out of the irradiated volume through the Si membrane. The Au stripe provides two additional channels for energy transport out of the grating area, namely due to the stripe's thermal conductivity and due to SPPs. The first contribution can be evaluated from the measured temperature gradients and amounts to $\sim 20 \%$ of the phonon transport through the $\mathrm{Si}$ membrane. The SPP contribution, which is determined in the next section as $5 \%$ of the incident power, is neglected at this point for reasons of simplicity. In this way we find an absorptivity (ratio of dissipated to incident power) of $19 \%$ and $5 \%$ for the polarisations in Fig. 2(a) and (b), respectively. The number for the p-polarisation is a lower limit because the energy transport by SPPs is not taken into account here. These values for the absorptivity are unaffected when the incident laser power is varied between 1 and $20 \mathrm{~mW}$. Such a behaviour is expected, because nonlinear effects should be negligible in this intensity range, as we demonstrate in Fig. S2 in the ESI. $\dagger$ In principle, part of the SPP intensity is also out-coupled into the far field as photons because the SPP modes are leaky. However, the energy loss associated with this effect is smaller than $1 \%$ of
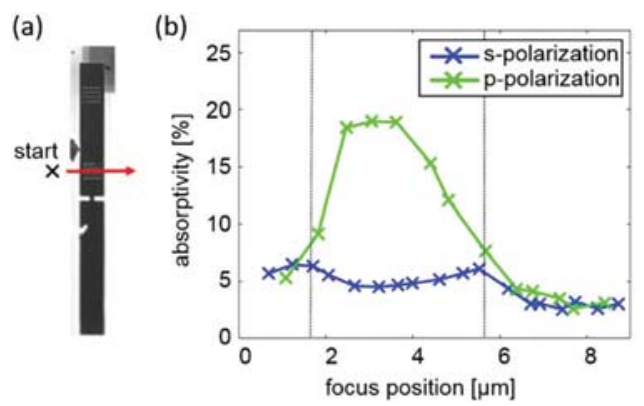

Fig. 3 Absorptivity of the plasmon grating, derived from the temperature increase of the Si membrane when the laser focus is scanned across the sample. (a) Path of the laser focus (red line), with the gold stripe in black. (b) Absorptivity for $\mathrm{p}$ - and s-polarised light as a function of the position of the laser spot. The dotted lines mark the edges of the gold stripe.

the total energy for samples fabricated by the same $\operatorname{method}^{27,36}$ and is therefore neglected in the current analysis.

The pronounced polarisation dependence of the coupling of the incident light into the grating is further illustrated in Fig. 3. In this case the laser focus was scanned across the grating, including a few $\mu \mathrm{m}$ on both sides outside the gold stripe. In Fig. 3(a) the path of the laser focus is sketched, and Fig. 3(b) shows the resulting absorptivity, again for both pand s-polarisation. Apparently, the data for the relative power dissipation agree outside of the grating. When the focus is on the grating, however, one observes again the quite distinct difference between the two polarisations.

How much of this additional optical power coupled into the grating for p-polarisation is transferred into propagating SPPs? - We first address this question from the theoretical side. Using the program Lumerical FDTD, ${ }^{42}$ which is based on a Finite Difference in the Time Domain (FDTD) scheme, one can determine the electric field distribution in and around objects like our grating, and in addition the dissipated power per volume element. Summing the latter over the whole volume and normalizing by the incident power then provides the absorptivity.

In Fig. 4 we show the spatial distribution of the normalised dissipated power density $P(x, y)$, locally averaged over the thick-

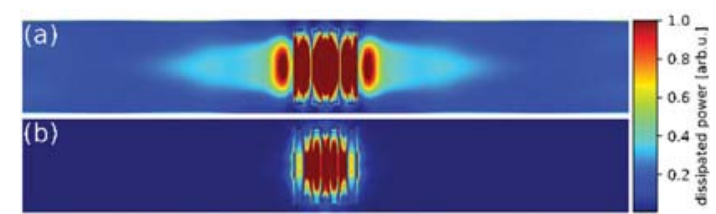

Fig. 4 FDTD simulation of the normalised dissipated power density with a monitor area extending over the whole gold stripe (width $4 \mu \mathrm{m}$ ). (a) p-polarisation and (b) s-polarisation. To increase the contrast for lower values, the colour code for the dissipated power density was capped at a threshold (identical for (a) and (b)) and normalized to that value. The uniform dark red regions in the centre of both (a) and (b) denote areas that are above that threshold. 
ness of the sample. Panel (a) was calculated for p-polarisation, where one expects resonant plasmon excitation. The dissipation is clearly enhanced compared to the bare optical absorption in panel (b) for s-polarisation. Integration over the dissipated power density in the upper panel gives a relative absorption of $18.2 \%$, in good agreement with the experimental result of $19 \pm 1 \%$ (Fig. 3(b), green curve). The same procedure leads the non-resonant absorption in the lower panel to a calculated absorptivity of $3.2 \%$, which is - as expected - considerably lower. The slight difference to the experimental value of $5 \pm$ 1\% (Fig. 3(b), blue curve) can be ascribed to the roughness in the grating structure, which leads to some enhancement of the coupling to light compared to the ideal grating assumed in the simulation. In the ESI we show (see Fig. S3†) that when limiting the monitor area to the grating itself, in the case of s-polarisation, not much changes. However, for p-polarisation only $12.3 \%$ absorptivity is found inside this smaller frame. So, according to the simulation, $68 \%$ of the absorbed power are dissipated directly in the grating, and $32 \%$ outside of the grating along the gold stripe. This part is carried by SPPs, which are generated in the grating and propagate along the gold stripe until they decay into phonons.

The additional heat dissipation outside the grating due to SPPs gives rise to an anisotropy in the temperature pattern, which, however, is weak under the steady-state conditions discussed so far, and is barely discernible in the experimental temperature map of Fig. 2(a). Likewise, finite element simulations (FE) with COMSOL Multiphysics, ${ }^{43}$ in which SPPs are included, exhibit only a slight plasmon-induced anisotropy in the steady state temperature landscape (Fig. 5 and S4 in the ESI $\dagger$ ). The propagating SPPs are implemented here as an additional, quasi-instantaneous heat source, distributed continuously along the gold stripe, with an exponential decay length of $12 \mu \mathrm{m}$, as known from other experiments on similar samples. ${ }^{27,37}$

The plasmon contribution should become much more explicit, if one made use of the fact that SPPs propagate with a velocity of nearly the speed of light, whereas thermal transport is much slower. The propagation of phonons is limited by the velocity of sound, and, moreover, for distances larger than the mean free path of the thermal phonons (several ten to
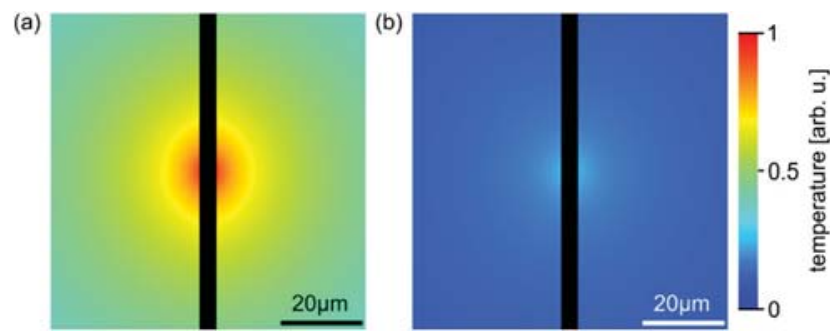

Fig. 5 FE simulations for steady state heating, calculated for similar conditions as in the experiment depicted in Fig. 2, (a) for p- and (b) for $\mathrm{s}$-polarisation. The slight anisotropy in the temperature distribution in (a) is caused by SPPs propagating along the gold stripe (width $4 \mu \mathrm{m}$, shown in black).
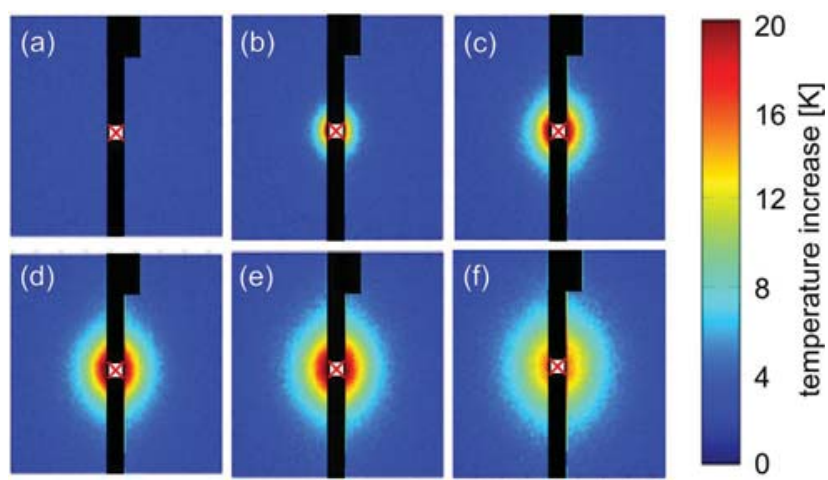

8

Fig. 6 Development of the temperature map for laser illumination with SPP generation, i.e. p-polarised light. The sample is different from the one in Fig. 2 and 3, but the dimensions of the gold stripe and the grating are the same. The time interval between subsequent panels is 330 ns. Panel (a): just before the laser was switched on; panel ( $f$ ): briefly after the laser was switched off, meaning that the centre is already cooling down, whereas further away from the centre the temperature is still rising. The size of each panel is $55 \times 55 \mu \mathrm{m}^{2}$.

hundred nanometres for our system at room temperature) the transport is diffusive and follows a $\sqrt{ } t$ law. This difference in the transport can be exploited in the dynamic measurements to which we turn now.

\section{Time-dependent measurements}

In Fig. 6 we show the temporal development of the experimental temperature map as the p-polarised laser pulse is switched on for a duration of $1.2 \mu \mathrm{s}$. The time interval between the panels is $330 \mathrm{~ns}$ (intermediate time frames taken at $110 \mathrm{~ns}$ intervals are shown in the ESI $\dagger$ ). In fact, not only can one see in this sequence that the steady state is approached here at a $\mu$ s scale, but also that the temperature pattern in this initial phase exhibits a clear deviation from radial symmetry, indicative of an additional heat source along the gold stripe, stemming from decaying SPPs.

For comparison we show in Fig. 7 a similar set of data, but this time for the sample rotated, such that the light polarisation is along the grating grooves, i.e., s-polarisation. As anticipated for this configuration - where no SPPs are excited - the temperature pattern is close to radially symmetric.

In Fig. 8 we have plotted the temperature development for several regions of interest (ROIs) along and perpendicular to the gold stripe, as they are marked in the schematic image in the insets. The curves in Fig. 8 confirm that the thermal relaxation time at the distances of these ROIs is around $1 \mu$ s for our sample, and they illustrate in Fig. 8(a) the contribution of the SPPs to the thermal dissipation. This contribution manifests itself in the difference for ROIs at the same distance from the grating, but in one case parallel and in the other case perpendicular to the gold stripe. Without the generation of SPPs (Fig. 8(b)), the dissipation in the grating is the only heat source, and therefore the curves for equivalent ROIs parallel and perpendicular to the gold stripe nearly coincide. Graphs complementary to Fig. 8, which show the spatial temperature 

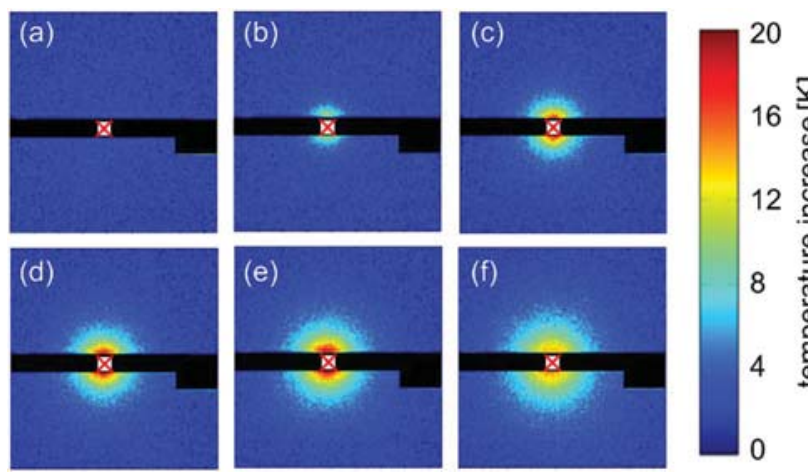

Fig. 7 Like Fig. 6, but for s-polarisation, i.e. without SPP generation. The change in the polarisation compared to Fig. 6 was in this case achieved by rotating the sample by $90^{\circ}$, in contrast to Fig. 2 and 3 , where the polarisation of the incident light was rotated. The incident laser intensity was increased here by a factor of 3 compared to Fig. 6 , in order to compensate partly for the smaller absorbance for this configuration and thus improve the signal-to-noise ratio.
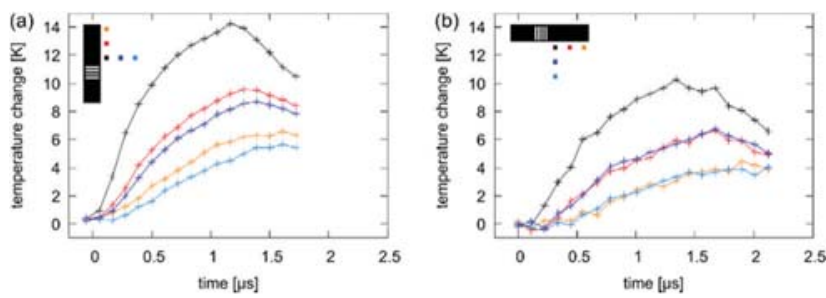

Fig. 8 Time dependence of the temperature increase after the onset of the laser pulse, for 5 positions on the Si membrane (ROIs). Graph (a) was recorded for illumination with p-polarised laser light, and graph (b) was for s-polarised light. The colours of the curves correspond to the colours of the ROls, whose positions are marked in the inset. The lines are guides to the eye.

profiles parallel and perpendicular to the gold stripe at $1 \mu \mathrm{s}$ after the beginning of the laser pulse, are presented in the ESI Fig. S5. $\dagger$

From the space and time dependence of the experimental data, one can obtain additional information by comparing them once again with FE simulations. The results are presented in Fig. 9 for both laser polarisations. In the simulations
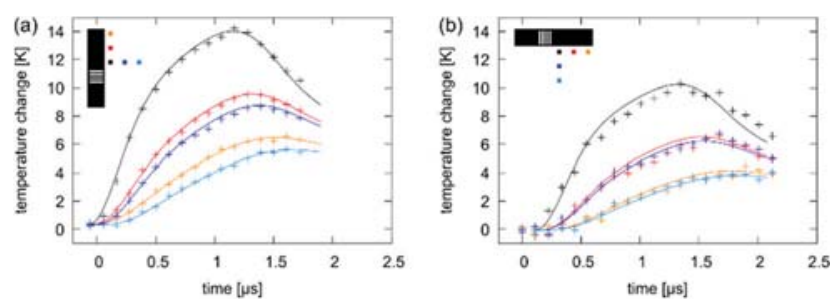

Fig. 9 Comparison with simulations: (a) p-polarised light and (b) $\mathrm{s}$-polarised light. The full lines show here the simulations for the optimum parameters indicated in the text $\left(\kappa / \kappa_{\text {bulk }}=0.45\right.$, fraction of SPPs $=25 \%$ ); the crosses are the experimental data from Fig. 8 . The fits with other parameter combinations are presented in the ESI. $\dagger$ two quantities were used as free parameters and determined for an optimal agreement with the experimental temperature traces: (i) the effective thermal conductivity of the Si membrane, which is known to be reduced compared to the bulk thermal conductivity of $\mathrm{Si}$ due to the small thickness of $340 \mathrm{~nm},{ }^{44}$ and (ii) the fraction of propagating SPPs with respect to the total dissipated power. In Fig. 9 we show only the results of the simulations for the optimum parameters; the results for some parameter range can be found in the ESI. $\dagger$ The best fit for the data in Fig. 9(b) (without SPP generation) is obtained for an effective conductivity $\kappa$ of our Si membrane which is reduced to $45 \%$ of the bulk thermal conductivity of $\mathrm{Si}, 148 \mathrm{~W}\left(\mathrm{~m} \mathrm{~K}^{-1}\right.$ at room temperature. This observation agrees well with the results in the literature. ${ }^{45}$

The same optimal value for $\kappa$ is found in Fig. 9(a), where in addition SPPs contribute as a heat source. As a second parameter we have in this case the fraction of propagating SPPs. In principle one could also use the decay length $l$ of the SPPs as an additional parameter to fit, but in order to keep the number of parameters as small as possible we have instead used the result $l=12 \mu \mathrm{m}$ measured independently for our type of sample. ${ }^{27}$ The best fit for the fraction of SPPs yields 25 $( \pm 5) \%$, which is below the (ideal) value of $32 \%$ derived from the FDTD simulations in Fig. 4, but still in reasonable agreement. Combining this finding with the absorptivity obtained in Fig. 3, one concludes that the overall efficiency for the generation of SPPs, propagating along one direction of the stripe, is $2.5 \%$ of the incident light intensity. This is lower than the best efficiencies of SPP generation reported in the literature, ${ }^{46}$ but one has to keep in mind that we have not systematically optimised our gratings, and also the role of the depth and roughness of the grating grooves, resulting from the FIB process as seen in Fig. 1(a), has not been explored.

\section{Conclusions}

The investigation of localised plasmons and propagating surface plasmon polaritons in nanoscopic and mesoscopic structures so far has mostly relied on direct optical detection, e.g. via evanescent or scattered light fields. We have presented here a method which also is all optical, but allows one to detect SPPs via their dissipation, utilizing the temperature dependence of the optical transmissivity of the thin Si membrane substrate. The generation of SPPs in a groove grating and their propagation along a thin gold stripe cannot only be traced, but the space- and time-dependent measurements of the temperature increase in the Si membrane supporting the stripe allow a quantitative comparison with simulations. From this analysis we determine both the resonant optical absorptivity of the grating and the fraction of SPPs which leave the grating area. The fraction of absorbed photons converted into SPPs is $25 \%$, and the intensity of SPPs travelling in one direction of the stripe is found to be $2.5 \%$ of the incident light intensity for the gratings used here. As a by-product, the investigation provides independently a value for the thermal con- 
ductivity of the Si membrane, which due to the small thickness is reduced compared to the bulk value by a factor of 0.45 . The stripe geometry used here is relatively simple; however, the technique is also applicable to more complex structures, and has promising potential for the investigation of hotspots, near-field enhancement, and general plasmon and heat management studies in nano-objects.

\section{Experimental}

The samples under study feature an Au stripe of $100 \mathrm{~nm}$ thickness and $4 \mu \mathrm{m}$ width evaporated on a free-standing Si membrane of thickness $340 \mathrm{~nm}$ and $700 \mu \mathrm{m} \times 700 \mu \mathrm{m}$ area supported by a massive Si wafer with a thickness of $\sim 500 \mu \mathrm{m}^{38}$ Finally, grooves were carved in the metal by FIB in order to form line gratings for the excitation of surface plasmons. The gratings are chosen to compensate the momentum mismatch between the SPPs and the exciting photons with wavelength $\lambda$ $=785 \mathrm{~nm}$ (ref. 27 and 37) and consist of 4 parallel grooves with $80 \mathrm{~nm}$ depth, $400 \mathrm{~nm}$ width and $3 \mu \mathrm{m}$ length, perpendicular to the stripe axis (see Fig. 1(a)). Furthermore, some of our samples have narrow constrictions in the Au stripe for studying the propagation of SPPs across nano-bridges, as described in ref. 27 and 37. This aspect, however, is not investigated in the present work.

The setup used for our experiments is shown in Fig. 1(b) and has been described previously. ${ }^{38,39}$ It consists mainly of an optical microscope which allows one to observe the optical transmissivity of the $\mathrm{Si}$ membrane samples with a high spatial resolution, a laser focused to $3 \mu \mathrm{m}$ diameter for the excitation of SPPs, and a pulsed light emitting diode which provides the illumination of the Si membrane. Since the transmissivity exhibits a pronounced temperature dependence, ${ }^{39}$ the lateral temperature distribution in the membrane can be inferred from the transmitted light, recorded by a digital camera.

\section{Conflicts of interest}

There are no conflicts to declare.

\section{Acknowledgements}

We thank H. Misawa, S. Juodkazis and all the SFB discussion group members for valuable and fruitful discussions on this work. We are grateful to M. Hagner for operating the lithography and focused ion beam system and for the valuable advice regarding sample preparation. We thank L. Kukk for technical support. We gratefully acknowledge the financial support from the Deutsche Forschungsgemeinschaft through SFB767 and the Strategic Japanese-German Cooperative Program of the JST and DFG on Nanoelectronics, from the Ministry of Science and Arts Baden-Württemberg through the Center of Applied Photonics, and from the Baden-Württemberg-
Stiftung in the framework of the Research Network Functional Nanostructures.

\section{Notes and references}

1 D. G. Cahill, P. V. Braun, G. Chen, D. R. Clarke, S. Fan, K. E. Goodson, P. Keblinski, W. P. King, G. D. Mahan, A. Majumdar, H. J. Maris, S. R. Phillpot, E. Pop and L. Shi, Appl. Phys. Rev., 2014, 1, 011305.

2 N. Li, J. Ren, L. Wang, G. Zhang, P. Hänggi and B. Li, Rev. Mod. Phys., 2012, 84, 1045.

3 Y. Dubi and M. Di Ventra, Rev. Mod. Phys., 2011, 83, 131.

4 S. Jezouin, F. D. Parmentier, A. Anthore, U. Gennser, A. Cavanna, Y. Jin and F. Pierre, Science, 2013, 342, 601.

5 L. Cui, W. Jeong, S. Hur, M. Matt, J. C. Klöckner, F. Pauly, P. Nielaba, J. C. Cuevas, E. Meyhofer and P. Reddy, Science, 2017, 355, 1192.

6 N. Mosso, U. Drechsler, F. Menges, P. Nirmalraj, S. Karg, H. Riel and B. Gotsmann, Nat. Nanotechnol., 2017, 12, 430.

7 R. W. Heeres, S. N. Dorenbos, B. Koene, G. S. Solomon, L. P. Kouwenhoven and V. Zwiller, Nano Lett., 2009, 10, 661.

8 J. T. Lü, M. Brandbyge and P. Hedegård, Nano Lett., 2010, 10, 1657.

9 D. Halbertal, M. Ben Shalom, A. Uri, K. Bagani, A. Y. Meltzer, I. Marcus, Y. Myasoedov, J. Birkbeck, L. S. Levitov, A. K. Geim and E. Zeldov, Science, 2017, 358, 1303.

10 J. Montgomery, T. N. Todorov and A. P. Sutton, J. Phys.: Condens. Matter, 2002, 14, 5377.

11 A. Pecchia, G. Romano and A. Di Carlo, Phys. Rev. B: Condens. Matter Mater. Phys., 2007, 75, 035401.

12 M. Galperin, K. Saito, A. V. Balatsky and A. Nitzan, Phys. Rev. B: Condens. Matter Mater. Phys., 2009, 80, 115427.

13 D. Dundas, E. J. McEniry and T. N. Todorov, Nat. Nanotechnol., 2009, 4, 99.

14 M. Tsutsui, S. Kurokawa and A. Sakai, Appl. Phys. Lett., 2007, 90, 133121.

15 W. Lee, K. Kim, W. Jeong, L. A. Zotti, F. Pauly, J. C. Cuevas and P. Reddy, Nature, 2012, 498, 209.

16 R. S. Sorbello, Solid State Phys., 1998, 51, 159.

17 R. H. M. Smit, C. Untiedt and J. M. van Ruitenbeek, Nanotechnology, 2004, 15, S472.

18 R. Berndt, R. Gaisch, J. Gimzewski, B. Reihl, R. Schlittler, W.-D. Schneider and M. Tschudy, Science, 1993, 262, 1425.

19 G. Schull, N. Néel, P. Johansson and R. Berndt, Phys. Rev. Lett., 2009, 102, 057401.

20 E. Ekici, P. Kapitza, C. A. Bobisch and R. Möller, Opt. Lett., 2017, 42, 4585-4588.

21 T. Lutz, C. Grosse, C. Dette, A. Kabakchiev, F. Schramm, M. Ruben, R. Gutzler, K. Kuhnke, U. Schlickum and K. Kern, Nano Lett., 2013, 13, 2846.

22 P.-J. Peters, F. Xu, K. Kaasbjerg, G. Rastelli, W. Belzig and R. Berndt, Phys. Rev. Lett., 2017, 119, 066803.

23 S. Berwerger, J. M. Atkin, R. L. Olmon and M. B. Raschke, J. Phys. Chem. Lett., 2012, 3, 945. 
24 C. Ropers, C. C. Neascu, T. Elsaesser, M. Albrecht, M. B. Raschke and C. Lienau, Nano Lett., 2007, 7, 2784.

25 M. Agio and A. Alù, Optical Antennas, Cambridge University Press, 2013.

26 M. D. Wissert, Optical Antennas: Linear and Nonlinear Excitation and Emission, KIT Scientific Publishing, Karlsruhe, 2012.

27 D. Benner, J. Boneberg, P. Nürnberger, G. Ghafoori, P. Leiderer and E. Scheer, New J. Phys., 2013, 15, 113014.

28 J. R. Krenn, M. Salerno, N. Felidj, B. Lamprecht, G. Schider, A. Leitner, F. R. Aussenegg, J. C. Weeber, A. Dereux and J. P. Goudonnet, J. Microsc., 2001, 202, 122.

29 B. Lamprecht, J. R. Krenn, G. Schider, H. Ditlbacher, M. Salerno, N. Felidj, A. Leitner, F. R. Aussenegg and J. C. Weeber, Appl. Phys. Lett., 2001, 79, 51.

30 R. Zia, J. A. Schuller and M. L. Brongersma, Phys. Rev. B: Condens. Matter Mater. Phys., 2006, 74, 165415.

31 C. Louis and O. Pluchery, Gold Nanoparticles for Physics, Chemistry and Biology, World Scientific, Singapore, 2nd edn, 2017.

32 D. R. Ward, F. Hüser, F. Pauly, J. C. Cuevas and D. Natelson, Nat. Nanotechnol., 2010, 5, 732.

33 Z. Ioffe, T. Shamai, A. Ophir, G. Noy, I. Yutsis, K. Kfir, O. Cheshnovsky and Y. Selzer, Nat. Nanotechnol., 2008, 3, 727.

34 N. Ittah and Y. Selzer, Nano Lett., 2011, 11, 529.
35 D. C. Guhr, D. Rettinger, J. Boneberg, A. Erbe, P. Leiderer and E. Scheer, Phys. Rev. Lett., 2007, 99, 086801.

36 D. Benner, J. Boneberg, P. Nurnberger, R. Waitz, P. Leiderer and E. Scheer, Nano Lett., 2014, 14, 5218.

37 C. Evans, P. Zolotavin, A. Alabastri, J. Yang, P. Nordlander and D. Natelson, Nano Lett., 2017, 17, 5646.

38 A. Ganser, D. Benner, R. Waitz, J. Boneberg, E. Scheer and P. Leiderer, Appl. Phys. Lett., 2014, 105, 191119.

39 M. Schmotz, P. Bookjans, E. Scheer and P. Leiderer, Rev. Sci. Instrum., 2010, 81, 114903.

40 R. Waitz, O. Schecker and E. Scheer, Rev. Sci. Instrum., 2008, 79, 093901.

41 M. Schmotz, J. Maier, E. Scheer and P. Leiderer, New J. Phys., 2011, 13, 113027.

42 Lumerical FDTD Solutions ( https://www.lumerical.com/).

43 Comsol Multiphysics ( https://www.comsol.de/).

44 H. J. Eichler, F. Massmann, E. Biselli, K. Richter, M. Glotz, L. Konetzke and X. Yang, Phys. Rev. B: Condens. Matter Mater. Phys., 1987, 36, 3247.

45 E. Chávez-Ángel, J. S. Reparaz, J. Gomis-Bresco, M. R. Wagner, J. Cuffe, B. Graczykowski, A. Shchepetov, H. Jiang, M. Prunnila, J. Ahopelto, F. Alzina and C. M. Sotomayor Torres, APL Mater., 2014, 2, 012113.

46 I. P. Radko, S. I. Bozhevolnyi, G. Brucoli, L. Martín-Moreno, F. J. García-Vidal and A. Boltasseva, Phys. Rev. B: Condens. Matter Mater. Phys., 2008, 78, 115115. 